\title{
GEO-SERVICIOS ELECTRÓNICOS DE LA ADMINISTRACIÓN PÚBLICA AUTONÓMICA Y LOCAL EN ARAGÓN (ESPAÑA) ${ }^{\star}$
}

\author{
Severino Escolano Utrilla ${ }^{1}$ \\ Departamento de Geografía y Ordenación del Territorio \\ Universidad de Zaragoza \\ severino@unizar.es \\ José Antonio Salvador Oliván ${ }^{1}$ \\ Departamento de Ciencias de la Documentacion e Historia de la Ciencia \\ Universidad de Zaragoza \\ jaso@unizar.es
}

\begin{abstract}
Resumen: Los geo servicios electrónicos se componen de utilidades y aplicaciones para analizar y visualizar información geográfica digital con objetivos específicos. Suelen estar diseñados para su utilización por usuarios finales. En Aragón, y en España, los geo servicios tienen una presencia escasa en todos los niveles de la administración pública.

En este artículo se describen y valoran algunos geo servicios públicos de la administración autonómica y local (Ayuntamiento de Zaragoza) y se propone un método para tipificar su fase de integración con otros servicios electrónicos y las capacidades de interacción con el usuario. El análisis llevado a cabo muestra que la mayor parte de los geo servicios públicos se limitan a presentar información de la localización de objetos y eventos, y son muy pocos los que realizan operaciones avanzadas de análisis espacial y permiten la interacción fluida con el usuario. La conclusión aboga por un mayor desarrollo de geo-servicios en áreas estratégicas como la seguridad, prevención de riesgos y otras, en el marco de la construcción de la administración electrónica, entendida ésta como una vía útil para la modernización de la administración pública.
\end{abstract}

Palabras clave: geo servicios, administración electrónica, información geográfica, Aragón.

* Recibido: 14-02-11. Aceptado: 07-07-11.

${ }^{1}$ Grupo de investigación GEOT. Universidad de Zaragoza. 
Abstract: Electronic geo-services are composed by a set of utilities and applications to analyze and display digital geographic information with specific purposes. They are usually designed to be used by end users. In Aragon, and in Spain as a whole, geo-services have a very weak presence at all levels of public administration.

Some public geo-services from autonomous and local government (Zaragoza City Council) are described and assessed in this article and a method is proposed to define their integration with other electronic services and their user interaction capabilities. The analysis carried out shows that the majority of public geo-services are limited to presenting information about the location of objects and events and very few perform advanced operations connected to spatial analysis and enable flexible user interaction. The conclusion supports greater development of geo-services in strategic areas like safety, risk prevention and others within the framework of the construction of an electronic administration, this being understood as a useful channel for the modernization of public administration.

Keywords: geo-services, e-government, geographical information, Aragon.

\section{Introducción}

La actuación del conglomerado de innovaciones tecnológicas, en particular las relacionadas con la información y comunicación digitales (TIC), se ha revelado, desde mediados de la década de 1970, como una de las principales fuerzas impulsoras de las transformaciones sociales y productivas que caracterizan a la sociedad del conocimiento.

Los impactos de las TIC son extensos y difusos, pues no se limitan a tal o cual actividad o aspecto de la vida socioeconómica o del ámbito privado de los individuos sino que, a través de un proceso de propagación capilar han llegado, con grados variables de intensidad, a todos los rincones de la sociedad y del territorio. Las TIC han posibilitado la renovación radical de procedimientos técnicos y modos de organización de la producción, los transportes, las comunicaciones, la prestación y adquisición de servicios y las actividades de consumo; también han contribuido a la recomposición de los equilibrios entre los agentes sociales y por tanto a la reestructuración de las relaciones de poder. 
La influencia de las TIC ha alcanzado también a la administración pública, no sólo en la reorganización de los procesos internos y en las formas del ejercicio de sus funciones, sino también en los modos de interacción con los ciudadanos en general, o con sectores socioeconómicos como las empresas, la educación, la seguridad, la sanidad o los servicios sociales, entre otros. Los gobiernos (Estados Unidos, Reino Unido, España), en todos sus niveles, han promovido programas para incorporar las TIC a las funciones y servicios de la administración, cuyas acciones se enmarcan en una emergente área de aplicación de las TIC denominada gobierno electrónico, e gobierno y a veces gobernanza electrónica, e gobernanza, (e government, e governance), aunque los dos conceptos no son sinónimos.

La administración pública ha sido, históricamente, el principal agente productor y de información geográfica y, al mismo tiempo, una usuaria masivas de dicha información con fines básicos de planificación, utilizando las tecnologías disponibles en cada época. La aparición y difusión de tecnologías digitales para la creación, gestión, distribución, análisis y visualización de información geográfica, han propiciado un salto cuantitativo y cualitativo en el valor y uso de esta clase de información. Si bien el empleo de información geográfica digital está bastante extendido, su difusión se halla limitada por las elevadas exigencias tecnológicas y de conocimiento especializado necesarios para su manejo. Ello no obstante, su empleo y consumo está progresando en el segmento de usuarios generales, desde los meros datos hasta los geo servicios, ofrecidos por empresas o prestados por la administración.

Por otro lado, los modelos de incorporación de las TIC en las empresas (e comercio por ejemplo) cuyo principal objetivo consiste en el incremento de la productividad, no son directamente trasplantables al sector público. Éste tiene características específicas derivadas de la naturaleza pública de sus funciones, de la necesidad de satisfacer múltiples propósitos, entre otros el de equidad social y territorial, y de la gran magnitud y complejidad de la organización de los Estados. Además, intervienen otros factores de tipo cultural o las inercias de las burocracias, la situación general del desarrollo socioeconómico, tecnológico etc., que hacen de la implantación de las TIC en la administración pública un proceso dificultoso y lento, que va mucho más allá de la creación de una página web.

En este contexto, el presente trabajo aborda el análisis, caracterización y valoración del uso de la información geográfica por la administración pública de la comunidad autónoma de Aragón, en el nivel del gobierno autonómico y en el municipal, este último ceñido al ayuntamiento de Zaragoza. También se defiende que el marco apropiado para su análisis el configurado por la administración electrónica (o gobierno lectrónico), y se propone un esquema metodológico para la valoración general de los geo servicios electrónicos. 


\section{Planteamiento del tema y objetivos: gobierno electrónico y geo servicios}

El despliegue de las TIC digitales en las tareas de la administración pública contiene un enorme potencial de transformación de los procesos de gobierno en todos sus niveles y dimensiones: política, técnica, económica, cultural y social, e incluso es un elemento básico de los enfoques, nacidos con el presente siglo, sobre la reforma del Estado.

La implantación de infraestructuras de las tecnologías, la disposición de nuevos métodos de organización, las modificaciones institucionales y culturales apropiadas para prestar servicios electrónicos de elevada calidad y valor a los ciudadanos, a las empresas y demás agentes sociales, conforman el ámbito de aplicación y de estudio del gobierno electrónico o e gobierno.

La Comisión Europea (Comisión Europea, 2003) define el e gobierno como "el uso de las tecnologías de la Información y las comunicaciones en las administraciones públicas, combinado con cambios organizativos y nuevas aptitudes, con el fin de mejorar los servicios públicos y los procesos democráticos y reforzar el apoyo a las políticas públicas; en el mismo documento se señala que "las tecnologías de la información y comunicación pueden ayudar a la Administración a enfrentar varios desafíos. No obstante, el núcleo no debe fijarse en las propias TIC. En su lugar, debe establecerse en el uso de las TIC combinado con cambios organizativos y nueva aptitudes para mejorar los servicios públicos, los procesos democráticos y las políticas públicas. Sobre esto trata el e-gobierno" (p. 4$)^{2}$.

Más recientemente, la propia Comisión Europea, en la página web sobre las tecnologías de la información y telecomunicaciones para el gobierno y los servicios públicos caracteriza el e gobierno del modo siguiente: "el e-gobierno consiste en el uso de herramientas y sistemas por las TIC para proporcionar mejores servicios públicos a los ciudadanos y a las empresas. Las TIC son utilizadas ya extensamente por departamentos de la administración así como por las empresas, pero el e-gobierno comprende más que las meras herramientas. El e gobierno eficaz significa también repensar la organización y los procesos, y cambiar el entorno para que los servicios públicos sean prestados de forma más eficiente a los ciudadanos que los necesitan. Bien implementado, el gobierno electrónico posibilita a todos los ciudadanos, empresas y organizaciones llevar a cabo sus relaciones con el gobierno de manera más fácil, más rápida y a menor coste"3. (http://ec.europa.eu/information_society/activities/ egovernment/index_en.htm).

\footnotetext{
${ }^{2}$ Traducción propia. Cursiva en el original.

${ }^{3}$ Traducción propia.
} 
El concepto gobierno electrónico sintetiza bien los múltiples aspectos comprendidos en la vía de la administración pública hacia la sociedad del conocimiento.

Uno de los ejes transversales más relevantes del e gobierno se identifica con la desmaterialización de la producción de servicios mediante la sustitución de tareas, materiales y personal por información y procedimientos digitales. Es obvio que esta metamorfosis acarrea importantes repercusiones en las dotaciones de infraestructura y de personal, así como en las modalidades de prestación de los servicios.

La información geográfica participa también en esta tendencia. La información geográfica, analógica y digital, ha sido tradicionalmente una materia prima estratégica para la planificación pública en general. La información geográfica digital ha llegado a los usuarios especializados básicamente en su forma elemental, como coordenadas que registran la localización de diversos fenómenos de interés. Sin embargo, los datos brutos se van incorporando a cadenas de procedimientos que añaden valor y conocimiento a la información geográfica, y que llegan al usuario final en forma de geo servicios.

\subsection{La construcción del gobierno electrónico}

Desde mediados de la década de 1990, la confluencia de las innovaciones y avances de las tecnologías de la información y las telecomunicaciones con las nuevas teorías de gestión nacidas en el sector empresarial, impulsó la aplicación de las TIC en tareas de la administración pública.

El debate sobre los modos y consecuencias de la implantación de las TIC en el sector público ha sido muy intenso y fecundo desde sus orígenes (Osborne y Gaebler, 1992; Tapscott, 1996), hasta tal punto que ha dado lugar a un campo específico dedicado al estudio científico de las esferas política, social y administrativa involucradas en la construcción del gobierno electrónico (Grönlund, 2004).

La práctica y los resultados de diversos trabajos de investigación han puesto de manifiesto que la incorporación de las TIC a la administración pública no consiste exclusivamente en un mero problema técnico, sino que abarca múltiples implicaciones propias de una forma diferente de producir, prestar y utilizar los servicios públicos. En grado variable, el uso de las TIC transforma la naturaleza del servicio, el qué de la función pública, y el cómo, cuándo y dónde se proporcionan los servicios (Grönlund, 2001) ${ }^{4}$.

\footnotetext{
${ }^{4}$ Véanse también las aportaciones fundamentales contenidas en las actas de las ocho conferencias internacionales sobre EGOV.
} 
En efecto, los servicios del e gobierno superan algunas restricciones respecto de los servicios tradicionales en cuanto a las condiciones de tiempo, espacio y forma. Los servicios virtuales se prestan durante 24 horas al día siete días a la semana, en cualquier lugar que disponga de infraestructuras técnicas apropiadas (habitualmente un ordenador conectado a la Internet) y se suministran de manera distinta, pues no es necesaria la presencia de un empleado público. También es posible acceder a cualquier servicio público desde un solo punto o portal (one-stop-government) (Wimmer, 2001; Traunmüller y Wimmer, 2005), lo que presupone la interconexión técnica de contenidos y operaciones de los diferentes departamentos de la administración.

El grado de madurez de los sistemas de gobierno electrónico suele establecerse mediante un modelo de fases sucesivas que se caracterizan a partir de la presencia creciente de dos componentes: la complejidad de los sistemas y de la tecnología utilizados, y el valor de los servicios prestados a los ciudadanos. El Plan de Administración Electrónica de Aragón (Gobierno de Aragón, s/fb) diferencia cuatro niveles en el proceso de desarrollo de la administración electrónica.

"Nivel 1. Información: Acceso a la información por diferentes canales

Nivel 2. Interacción: Búsqueda de información; acceso a descargas de documentos (formularios, estadísticas, normativa, etc.)

Nivel 3. Transacción: Inicio y finalización de trámites (autenticación de usuarios, procesos de negocio integrados en los diferentes canales

Nivel 4. Integración: Intercambio electrónico automático de información y transacciones entre Administraciones; Sistemas de back office integrados, reingeniería de procesos".

Estos escalones y sus contenidos coinciden con los cuatro "estadios críticos" de la evolución del gobierno que distingue Bhatnagar (2004): presencia de páginas web: los departamentos facilitan a los ciudadanos información sobre procedimientos); integración limitada: los departamentos se relacionan entre sí por intranet, que permite la comunicación mediante correo electrónico, las consultas de bases de datos y la descarga de formularios; transacciones: los servicios electrónicos están total o parcialmente automatizados, las aplicaciones incluyen certificaciones y licencias; transformación: todas las etapas de las transacciones del gobierno, incluidos los pagos, se realizan por vía electrónica, implementadas en nuevos modelos de prestación de servicios en los que participan empresas privadas.

La implementación de las TIC en la administración pública registra avances considerables en los países desarrollados y en vías de desarrollo aunque, al mismo tiempo, plantea dificultades de variada naturaleza (Bhatnagar, 2004; Millard, Shahin et al., 2009). Fountain (2001) reconoce que las tecnologías de la información pueden cam- 
biar las estructuras de las organizaciones privadas y públicas, y pone de manifiesto los problemas institucionales y de gobernabilidad asociados al uso de las TIC y su interacción con las estructuras del Estado. Wimmer et al. (2001) ya advirtieron tempranamente que la implantación directa de los modelos de incorporación de las TIC a las actividades de las empresas (e business) al gobierno virtual no es viable, aunque aquéllos puedan servir de estímulo y de inspiración en algunos aspectos. Por otro lado, Rivera (2003) da cuenta de las complejas relaciones entre la incorporación de las TIC en la administración pública y las estructuras económicas de los países, que ejemplifica en el caso chileno.

Desde el punto de vista operativo también se presentan barreras a la difusión de las TIC en la administración pública, como la insuficiencia de las dotaciones de infraestructuras o las carencias de formación técnica de los usuarios y de los empleados públicos. En todo caso, persisten desafíos adicionales en la construcción del gobierno que trascienden las metas de mayor eficiencia (Wimmer, Traunmüller et al., 2001), que se relacionan con la cultura y las estructuras sociales (Garson, 2000), con la función reguladora del Estado, con la equidad social y territorial y con la transparencia y responsabilidad propia de regímenes democráticos. El gobierno electrónico es un medio útil para acometer reformas y mejorar la eficiencia en el sector público, pero el modo alguno es la solución única para este fin.

\subsection{Información geográfica y geo servicios}

Buena parte de las actividades diarias de las personas son de naturaleza geográfica ya que su contenido se relaciona con la localización en que se desarrollan. Asimismo, la organización y el funcionamiento de los mercados revelan estructuras espaciales decisivas, a veces, en los precios de los productos y en la calidad de los servicios. Por estas y otras razones no es extraño, pues, que la administración pública utilice masivamente información y consideraciones geográficas en los procesos de planificación y de toma de decisiones de todo tipo para definir y resolver problemas más o menos urgentes.

La información geográfica no sólo es útil para comprender y caracterizar fenómenos del mundo real, sino también como referencia para organizar y analizar cualquier información a partir de una cualidad fundamental: la localización.

Los modelos de datos de información geográfica son más complejos que la mayoría de los modelos de datos, ya que el datum de información geográfica (una unidad significativa de información) combina de manera indisoluble información temática, espacial y, opcionalmente, temporal. Así pues, la elaboración, gestión y análisis de 
información geográfica requiere cierto nivel de equipamiento técnico y de conocimiento especializado.

Los mapas en papel han sido tradicionalmente el modo privilegiado de representar información geográfica, y se han utilizado para registrar, visualizar y analizar esta clase de información. La creación de bases de datos geográficos digitales junto con la disposición de programas informáticos específicos para su análisis y modelado (especialmente los sistemas de información geográfica, SIG) han multiplicado las posibilidades de análisis y visualización de la información geográfica, y han permitido su difusión en áreas especializadas y entre usuarios en general. Es más, los SIG constituyen un marco general útil, tanto en las empresas como en la administración pública, para organizar, analizar, visualizar y distribuir todo tipo de datos necesarios para resolver problemas, mejorar procesos, desarrollar planes y definir políticas.

Los geo servicios electrónicos se componen de información geográfica y utilidades y aplicaciones para la gestión, análisis y visualización de la misma y de los resultados, con un propósito determinado, como obtener la ruta más corta entre dos localizaciones o seleccionar eventos a una distancia dada de un objeto.

Un rasgo esencial de los geo servicios es su orientación hacia usuarios finales que, en su mayor parte, no poseen conocimientos avanzados en el manejo de la información geográfica. Ello implica que las interfaces y procedimientos han de diseñarse para que sean inteligibles a usuarios no expertos en SIG. Por otro lado, esta condición ha podido limitar la difusión de los geo servicios basados en Internet.

Gran parte de los servicios relacionados con la información geográfica se encuadran en el nivel 1 del modelo antes citado y consisten en la simple distribución de datos. Sin embargo, los avances en la creación de estándares de datos (OPEN GIS) y de infraestructuras de datos espaciales (IDE), el incremento de la capacidad de transmisión de las redes, las mejoras de las interfaces (Web 2.0) y otros factores, han facilitado la integración y colaboración entre usuarios y productores de datos y servicios; en otras palabras: han allanado el camino hacia la creación de geo servicios. Estos y otros avances posibilitan, al menos sobre el papel, la aplicación de funciones de los SIG en cualquier ordenador personal y en dispositivos móviles 5 .

Algunas empresas ofrecen determinados servicios digitales de complejidad variable que abarcan desde la localización de objetos, actividades y de direcciones postales hasta el control de flotas de automóviles, camiones o autobuses; la búsqueda de rutas entre varios puntos, o de la mejor combinación de medios de transporte urbano

\footnotetext{
${ }^{5}$ Los geo-servicios basados en Internet de cierta envergadura suelen organizarse en un servidor central que coordina tareas SIG realizadas por ordenadores conectados en red (por ejemplo los geo-servicios pueden integrar funciones de análisis espacial, tan populares como las de Google Maps).
} 
para desplazarse entre varios destinos que satisface algún criterio (número mínimo de trasbordos, recorrido más corto); la organización de diversos servicios de emergencia; el seguimiento en tiempo real de animales anillados para conocer sus patrones de desplazamiento, y otros.

Los diferentes niveles de la administración pública también prestan geo servicios digitales, si bien los de carácter integrado (estadios 3 y 4 del modelo referido) son muy escasos, pues necesitan elevadas inversiones en datos e infraestructura, los gastos de personal suelen ser considerables, y precisan una organización que permita el flujo de información entre diferentes departamentos. Por otro lado, ciertas determinaciones legales, como las que afectan al secreto estadístico, pueden limitar también el alcance de algunos de los servicios digitales. Por estas razones, al menos en España, la mayor parte de los geo servicios públicos digitales son proporcionados por los institutos o servicios encargados de la creación y mantenimiento de información geográfica; son más escasos, en cambio, los desarrollados por otras instituciones (ayuntamientos, por ejemplo) o departamentos y relacionados con el catastro, la seguridad, la salud, la gestión de emergencias o del medio ambiente.

\subsection{Objetivos}

El desarrollo de geo-servicios en la administración pública se encuentra en una fase incipiente. Tanto es así, que en el ámbito de estudio científico del e gobierno no se registran contribuciones sobre el tema, ni en el mismo participan especialistas procedentes de las disciplinas geográficas o espaciales. En una investigación reciente en la que se analizan 3.281 publicaciones sobre e gobierno correspondientes a 3.506 autores, no se recoge ningún autor de campos disciplinares relacionados con las ciencias geográficas o espaciales; un área temática próxima podría ser la de "ciencias sociales", en la que se enmarcan tan sólo el 2\% de las contribuciones (Scholl, 2009).

En consecuencia, parece oportuno llevar a cabo un estudio para caracterizar la naturaleza de los geo servicios electrónicos prestados a escala autonómica y local, ya que estos niveles administrativos poseen la mayor parte de las competencias de los servicios públicos que más afectan a la vida diaria de los ciudadanos.

El presente trabajo tiene dos objetivos principales interrelacionados: a) analizar y valorar los geo servicios del gobierno de Aragón del Ayuntamiento de Zaragoza en el contexto presentado, en materia de las funciones que prestan, su organización, magnitud, su etapa de desarrollo, uso actual y potencial así como por su contribución a la construcción del e gobierno; b) proponer y ensayar un método útil para explorar y valorar la contribución de los geo servicios a la implantación de la administración electrónica a escala local. 


\section{Metodología y datos}

Un marco metodológico adecuado para la valoración y análisis de los geo sevicios se puede construir a partir de la estructura de las unidades funcionales y de los flujos de información que configuran un geo-servicio, como se representan en el esquema de la figura 1.

De cada uno de los componentes diferenciados analizan sus dimensiones significativas a través de las variables e indicadores que las definen y se estima su complejidad y fase de evolución en cuanto a la integración con otros servicios de administración electrónica. Además se consideran otros aspectos básicos relativos al conjunto del geo servicio, como la organización técnica y funcional, el personal que lo gestiona, y las infraestructuras y tecnología utilizadas.

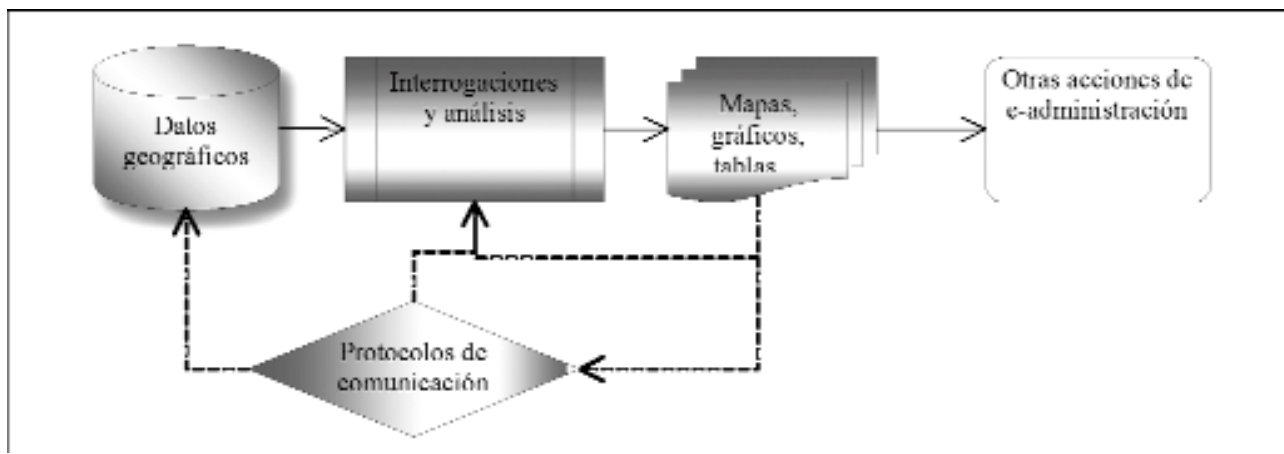

Figura 1. Esquema de las unidades y flujo de información de un geo servicio.

Los conceptos y categorías mencionados se organizan en una tabla de doble entrada que facilita la toma de los datos y futuros análisis. La evolución y estado de madurez de un geo servicio se puede tipificar a partir de un modelo de etapas como el mencionado con anterioridad; Esteves (2006) ha empleado una versión equivalente, así como Chaín y Tomás (2005) que también han desarrollado un esquema operativo equivalente para propósitos de evaluación. En ambos casos se distingue cinco etapas en el desarrollo del gobierno electrónico: 1) información (información pública en la página web); 2) comunicación unidireccional (descarga de instrucciones y otros documentos); 3) comunicación bidireccional (relleno de formularios, realización de reservas); 4) transacciones e interacción de datos (trámites completo); 5) información y conocimiento intra adaministraciones (integración de todos los servicios de administración electrónica). 
La información se ha recogido a partir de la revisión sistemática de los contenidos de los geo servicios publicados en las correspondientes páginas web páginas web del portal de centros educativos de la DGA y del Ayuntamiento de Zaragoza.

Por otra parte, se han llevado a cabo entrevistas con los responsables y técnicos de los departamentos en los que se encuadran los geo servicios para recabar información cualitativa y opiniones acerca de la organización del servicio, sus costes, dificultades de mantenimiento, políticas de desarrollo y de uso y, en fin, sobre la utilidad general del geo servicio ${ }^{6}$.

\section{Resultados y valoración}

En los países desarrollados la modernización de la administración pública y la mejora de los servicios que presta pasan, en gran medida, por la integración de las TIC en los procesos de creación y suministro de servicios a los ciudadanos, a las empresas y a otras organizaciones sociales.

Esta estrategia se sigue de forma decidida en la Unión Europea, aunque con ritmos diferentes en cada estado, acordes con su grado de desarrollo económico general y otros factores. En todos los niveles de la organización política de la UE se han aprobado planes para impulsar y ordenar el despliegue de las TIC en la administración pública, inscritos en el marco de funcionamiento de la UE y de los derechos y deberes de los ciudadanos.

En España, se ha aprobado un conjunto de normas de rango diverso que regulan el desarrollo y funcionamiento de los servicios electrónicos prestados por las diferentes administraciones públicas. En la administración del Estado se observan grandes diferencias en el grado de implantación de servicios electrónicos entre algunos ministerios y departamentos en los que se hallan bien establecidos (Hacienda) y la mayoría, en los que apenas están presentes. En los ayuntamientos el panorama es similar: en la mayoría no existen estos servicios o se prestan de forma aislada y con niveles de eficiencia bajos (Esteves 2006).

La Ley 11/2007, de 22 junio, de acceso electrónico de los ciudadanos a los servicios públicos, reconoce una serie de derechos para los ciudadanos y las consiguientes obli-

\footnotetext{
${ }^{6}$ Agradecemos a los responsables y técnicos del Centro de Información Territorial de Aragón, de varias direcciones generales del Departamento de Educación, Cultura y Deporte del Gobierno de Aragón, y de la Unidad de Gestión de la web municipal del Ayuntamiento de Zaragoza, la información que nos han facilitado y el tiempo que nos han dedicado en las entrevistas que hemos mantenido con ellos.
} 
gaciones para las administraciones públicas en relación a las formas de uso y prestación de los servicios públicos; entre los más importantes de los primeros cabe destacar:

Derecho a relacionarse con las Administraciones Publicas utilizando medios electrónicos para acceder a información y realizar consultas y llevar a cabo otros tipos de actos administrativos a través de alguno de los canales disponibles.

Derecho a no presentar datos y documentos en poder de la Administración.

Derecho a conocer por medios electrónicos el estado de tramitación de los expedientes que les afecten y obtener copias electrónicas de documentos.

Derecho a obtener los medios de identificación electrónica necesarios; las personas físicas pueden utilizar los sistemas de firma electrónica del DNI

Por otra parte, el reciente $\mathrm{RD} 4 / 2010$, de 8 de enero, que regula el Esquema Nacional de Interoperabilidad, establece los criterios y recomendaciones de seguridad, normalización y conservación de la información, de los formatos y de las aplicaciones que deberán ser tenidos en cuenta por las Administraciones públicas para asegurar un adecuado nivel de interoperabilidad organizativa, semántica y técnica de los datos, informaciones y servicios que gestionen en el ejercicio de sus competencias y para evitar la discriminación a los ciudadanos por razón de su elección tecnológica.

Finalmente, las disposiciones aprobadas para trasponer la Directiva 2006/123/CE del Parlamento Europeo y del Consejo, relativa los servicios en el mercado interior, componen un nuevo marco jurídico y administrativo que ordena la prestación de los servicios entre las empresas y ciudadanos de los estados de la UE. Esta normativa pretende eliminar los obstáculos que se oponen a la libertad de establecimiento de empresas de servicios y circulación de los servicios entre los países de la UE, para convertir, a partir de 2010, un mercado fragmentado en otro mercado interior de servicios único y efectivo.

La aplicación de esta directiva y de otra normativa relacionada con la administración electrónica, comporta, entre otras acciones, la construcción de un portal único por cada país y comunidad autónoma, la normalización de los procedimientos y la interoperabilidad entre los diferentes portales de servicios electrónicos de las administraciones públicas de la UE.

\subsection{El marco del desarrollo de los servicios públicos electrónico en Aragón}

Desde la formación de la Comunidad Autónoma de Aragón (1978) los responsables de su gobierno han sido conscientes de la conveniencia de disponer de una 
administración pública moderna lo que comporta, entre otros aspectos, la necesaria incorporación a la misma de las TIC.

En la práctica, las intenciones se han traducido en diversas acciones, no siempre bien coordinadas, encaminadas a la mejora del equipamiento informático, al tratamiento automático de datos, a la creación de la red aragonesa de comunicaciones institucionales (RACI), a la creación de la entidad pública aragonesa de Servicios Telemáticos (2001) encargada de la gestión de los sistemas, servicios y aplicaciones de telecomunicación e informática de la administración pública aragonesa, entre otras de menor alcance.

Con el fin de impulsar y coordinar acciones para el uso eficiente de las TIC en la administración pública, el gobierno de Aragón ha puesto en marcha varios planes e iniciativas. Los más importantes son el Plan de Administración Electrónica del Gobierno de Aragón", el "Plan Localidad" y el "Plan Tecnológico para el desarrollo de la administración electrónica en Aragón"; estos tres planes "constituyen un completo marco para el desarrollo de la administración electrónica en la Región” (Gobierno de Aragón, s/f c, 88).

El Plan de Administración Electrónica (PAE) “...se constituye como el eje fundamental, hoja de ruta, de la modernización de la Administración de la Comunidad Autónoma durante los próximos años y, en consecuencia, está integrado por la relación de iniciativas, proyectos técnicos, desarrollos normativos, así como el resto de actuaciones que se ha considerado necesario llevar a cabo en el horizonte temporal previsto" (Gobierno de Aragón, s/f b: 4).

El Plan de Administración Electrónica establece cuatro ejes en torno a los cuales se articulan las políticas, proyectos e iniciativas que contempla, así como el análisis y diagnóstico de la situación actual: e servicios, e gestión, e infraestructuras y e gobernanta. El eje de los e servicios está conformado por "todas aquellas acciones, medidas proyectos y/o iniciativas especialmente destinadas a la creación y definición de las relaciones externas y servicios que el Gobierno de Aragón ofrece a los ciudadanos, las empresas y el resto de entidades en materia de administración electrónica" (Gobierno de Aragón, s/f b: 17).

En el "modelo técnico de prestación de servicios de Administración Electrónica" del PAE se considera el "servicio de información geo referenciada" un "servicio de uso común" (junto, por ejemplo, con el de firma electrónica o el de gestión documental) (Gobierno de Aragón, s/f b: 19). Esta importancia deriva del hecho de que la información geográfica es un elemento fundamental para la prestación de servicios electrónicos, e imprescindible para la elaboración de geo-servicios.

El Centro de Información Territorial de Aragón es el servicio público encargado de crear y mantener la información geográfica del territorio de la Comunidad 
Autónoma. La actividad de este servicio, en particular la relativa a la de obtención y gestión de información geográfica se reglamentará en un decreto del gobierno de Aragón que ya ha pasado la fase de exposición pública. En términos generales se puede afirmar que este servicio ha puesto a disposición de los ciudadanos, las empresas y la administración, un cuerpo de información geográfica de Aragón actualizada y accesible. Los datos se han organizado en un Sistema de Información Territorial de Aragón (SITAR) que facilita el manejo de la información y la descarga de archivos. Además, este Centro presta asistencia técnica a otros departamentos de la administración en materia de uso de información geográfica, para lo que dispone de personal bien cualificado y de recurso tecnológicos suficientes (figura 2).

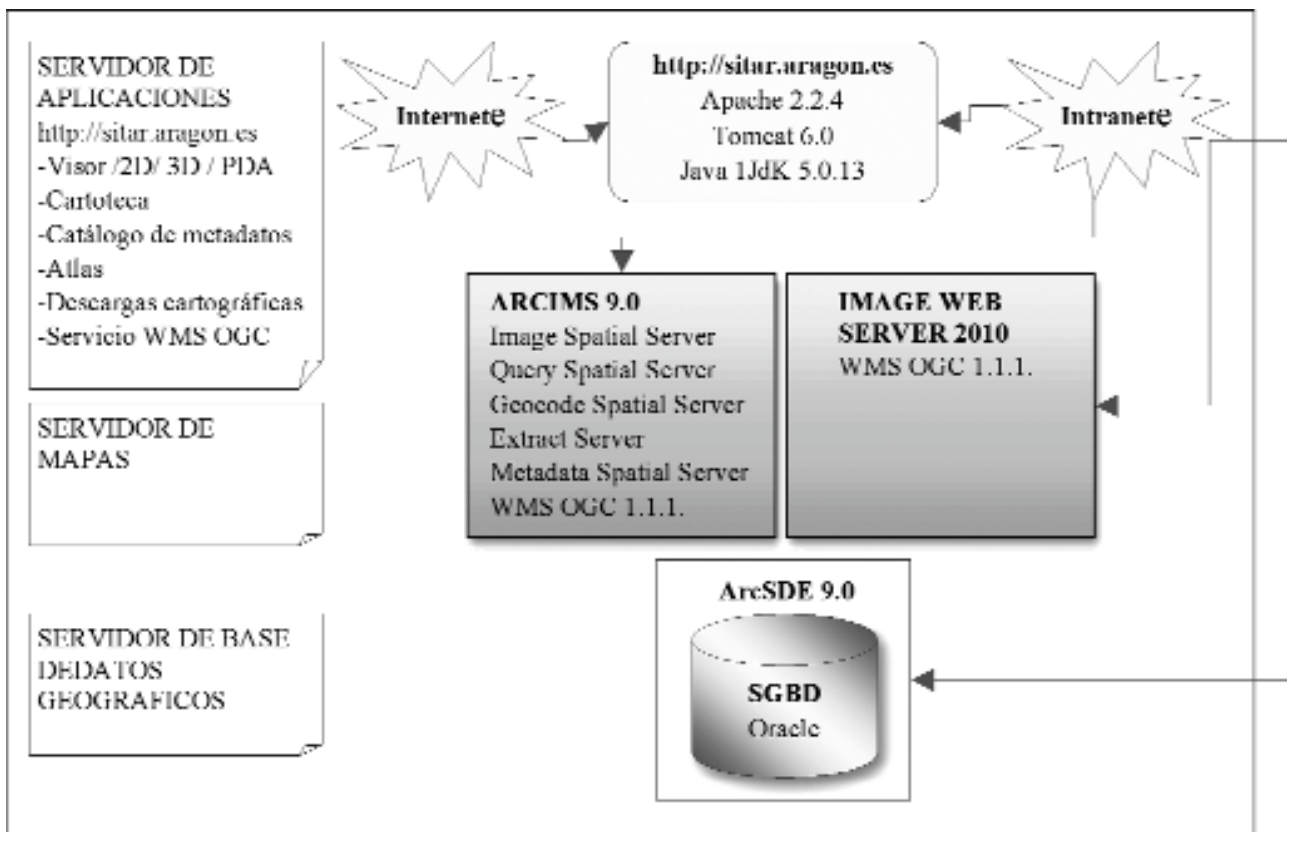

Figura 2. Estructura funcional y tecnología del SITAR.

Fuente: Centro de Información Territorial de Aragón.

El Ayuntamiento de Zaragoza inició, en 2004, el proyecto de "Infraestructura de Datos Espaciales del Ayuntamiento de Zaragoza: IDEZar", para crear un cuerpo de información geográfica digital sobre el término municipal, y ponerla a disposición de sus propios departamentos, las empresas y del público en general. Aparte de este objetivo fundamental, el desarrollo de IDEZar tiene implicaciones positivas importantes en materia de difusión de las tecnologías de la información entre los ciudadanos y la propia gestión municipal, a la vez proporciona información fundamental para 
la planificación y ejecución de políticas municipales, especialmente en materia de medio ambiente, movilidad, cultura, y para diversos objetivos de las empresas y usuarios particulares.

IDEZar se enmarca en la iniciativa INSPIRE ("INfraestructure for SPatial InfoRmation in Europe") (European Commission INSPIRE) de la que constituye un nodo local. Esta relación exige que la información geográfica sea estructurada de acuerdo a diversos estándares lo cual facilita su difusión y uso por varios sistemas (Internet, teléfono móvil). Actualmente, el sistema contiene un considerable volumen de información geográfica sobre aspectos del medio físico y de abundantes clases de elementos propios de la actividad socioeconómica del término municipal de Zaragoza y permite algunas conexiones funcionales con otros nodos de la red. Para seleccionar, analizar y visualizar la información geográfica se han programado varias utilidades que proporcionan al usuario opciones de búsqueda muy flexibles y un grado avanzado de interacción con el sistema (Ayuntamiento de Zaragoza-Web Municipal, 2010). La gran magnitud de la información de IDEZar y loas variadas funciones de búsqueda, análisis y visualización hacen que este sistema adquiera un alto grado de complejidad tecnológica y de organización (Portolés-Rodríguez, Álvarez et al., 2005; Álvarez, Muro et al,. 2006).

En consecuencia, estas dos grandes geo bases de la administración pública, y otras públicas (por ejemplo: CartoCiudad (Mas, García et al., 2010)) y privadas proporcionan la información geográfica fundamental para elaborar geo servicios electrónicos en Aragón.

El Gobierno de Aragón presta ya diversos servicios electrónicos y otros están planificados o en desarrollo: información al ciudadano (portal del gobierno de Aragón, publicaciones oficiales, catálogos de procedimientos), registro telemático, notificaciones telemáticas, servicios de respuesta inmediata, pasarela de pagos, plataforma de firma electrónica, gestión de representantes ${ }^{7}$. Otras entidades de la administración local también tienen implementados diversos servicios electrónicos relacionados con sus funciones, como la diputación de Zaragoza y varios ayuntamientos.

Sin embargo, a pesar de la importancia clave que tiene la información espacial en la arquitectura de la administración electrónica, apenas existen geo servicios públicos desarrollados en torno a la información geográfica, ni en el nivel autonómico ni en el local. Los que se describen en el apartado siguiente son los únicos accesibles al público a través de Internet.

Por otro lado, la dispersión geográfica de la población y aragonesa es un aspecto que, potencialmente, dificulta o encarece la creación de infraestructuras y la presta-

\footnotetext{
${ }^{7}$ http://portal.aragon.es/portal/page/portal/DGA/ENLINEA
} 
ción de servicios electrónicos. Buena parte del territorio sólo tiene acceso a una oferta limitada de infraestructuras y servicios; así en 2005, 226 municipios (el 31\%) carecían de servicios de ADSL y 219 (30\%) sólo disponían de estos servicios parcialmente (Gobierno de Aragón, s/f a, 11). Los telecentros (figura 3), si bien pueden ser una solución para acceder a contenidos de Internet, no son las instalaciones adecuadas para el uso de geo servicios debido a su propia naturaleza (estar ubicados en edificios públicos, horarios de uso limitados, etc.).

\subsection{Geo servicio: Buscador de centros educativos del Gobierno de Aragón}

El Buscador de centros Educativos del Gobierno de Aragón (Gobierno de Aragón 2010) está concebido como un geo servicio para ayudar a los usuarios a elegir centros de enseñanza no universitaria en la ciudad de Zaragoza en función de determinadas características de los mismos, y para calcular la puntuación obtenida en la solicitud de elección de un determinado centro.

La creación de este geo servicio aborda, además, otros retos que debe afrontar la administración educativa, como proporcionar una plataforma única para intereses de los agentes involucrados en la educación (Consejo Escolar, sindicatos, Justicia de Aragón...), reducir la documentación que genera la gestión de los procesos educativos, disponer de un sistema capaz de simular las demandas de los centros para facilitar la planificación y, en fin, incorporar la distancia entre el domicilio de los alumnos y los centros escolares como un criterio para la adjudicación de centro escolar (Faci, 2010).

La proximidad del domicilio o del lugar de trabajo de los padres es uno de los criterios básicos para admisión de estudiantes en los centros escolares, hasta el punto de que más del 90\% de las familias eligen centros cercanos al domicilio familiar (Faci, 2010: 9). El modo tradicional de asignar un valor a la proximidad ha consistido en zonificar el término municipal y dar prioridad a los residentes de cada zona en los centros comprendidos en la misma. Con esta solución es posible que se asignen centros de la zona de un domicilio determinado, más alejados que otros centros de otra zona contigua; esta circunstancia es frecuente en las áreas adyacentes a los límites de las zonas.

Con la aplicación de la tecnología de los sistemas de información geográfica y de los sistemas de posicionamiento global (GPS), es posible obtener la distancia que separa un domicilio o un lugar de trabajo de un centro educativo y considerarla como un elemento de baremación para la elección de un centro. La distancia, calculada por el Sistema de Información Territorial de Aragón (SITAR), se mide en línea recta, y se 


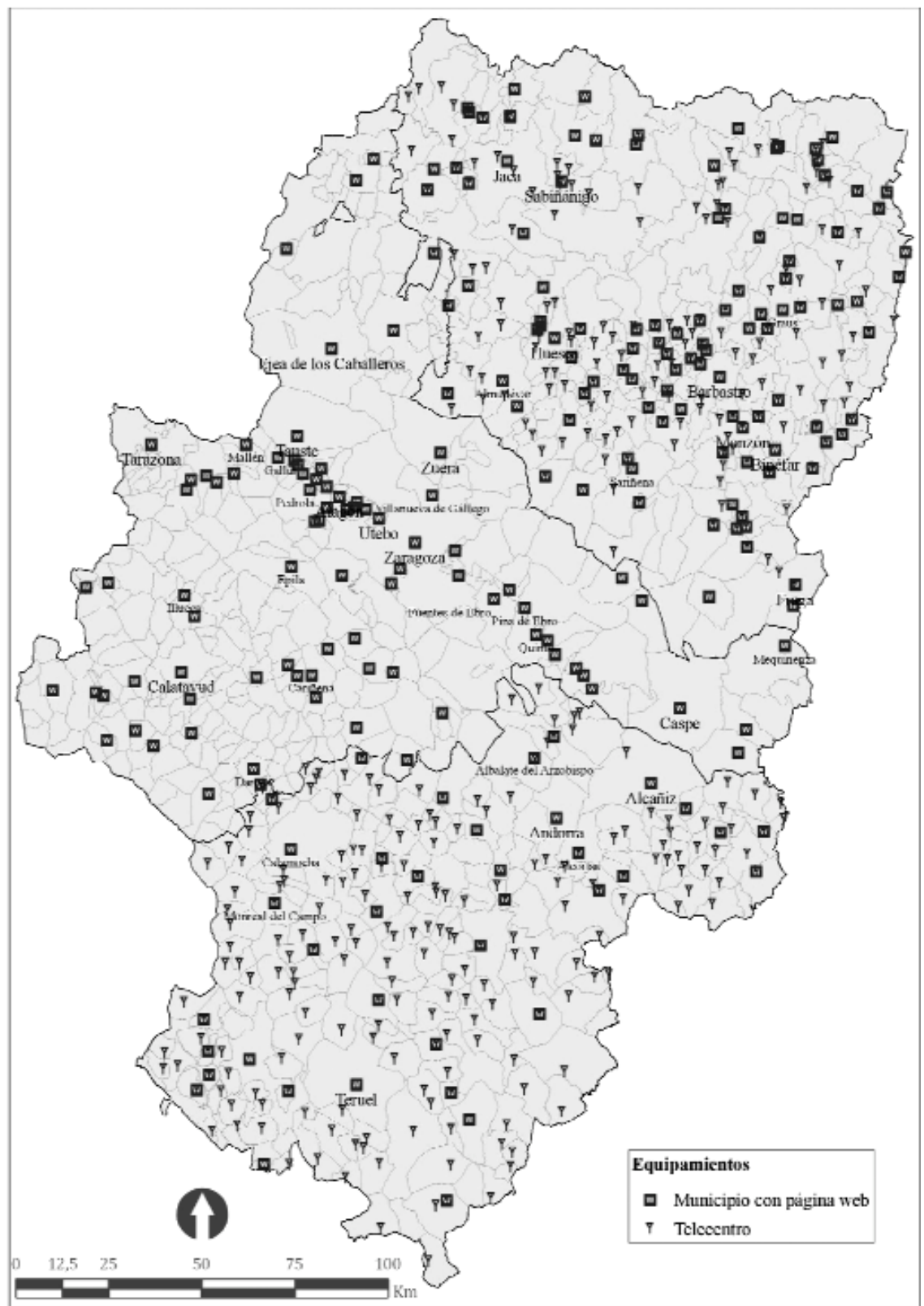

Figura. 3. Ayuntamientos con página web y red de telecentros en Aragón. 2010.

Fuente: Red.es, para los telecentros, Observatorio Aragonés de Sociedad de la Infomación, para las páginas web. Elaboración propia. 
puede ponderar en función del tipo de suelo (por ejemplo: un parque; una avenida) que separa los puntos que se relacionan (centroides de parcelas catastrales). Por otro lado, se pueden establecer distancias mínimas (y máximas) para tener en cuenta este criterio en las solicitudes.

Por otra parte, el geo servicio dispone de herramientas complementarias que facilitan la búsqueda de centros según diversos criterios y la simulación de los baremos teniendo en cuenta la proximidad... Los usuarios acceden a la aplicación través del portal de centros educativos en el que existen diferentes interfaces de búsqueda en las que el usuario puede introducir diferentes parámetros y criterios de búsqueda. Otras características más importantes de este geo servicio aparecen en la tabla 1.

Este geo servicio ha permitido incorporar un elemento fundamental al proceso de elección de centro escolar, como es la distancia desde domicilio de los estudiantes, o centro de trabajo de los padres, al centro escolar, de forma eficaz y transparente. Por estas y otras razones este geo servicio ha recibido, entre enero y octubre de 2010, más de 150.000 visitas.

La organización de la tecnología y de las infraestructuras del buscador de centros educativos reviste cierta complejidad, pues tanto los servidores de información como la gestión se hallan distribuidos entre servicios de varios departamentos del Gobierno de Aragón. Por una parte, el Departamento de Educación produce los datos correspondientes a los centros y zonas de escolarización y, por otra, el Centro de Información Territorial de Aragón mantiene la información espacial correspondiente tanto a la localización de los centros escolares, zonas de escolarización, domicilios y tipos de suelo, y lleva a cabo las operaciones de análisis espacial.

Desde el punto de vista del usuario, el buscador de centros educativos sigue una lógica sencilla: selección de centros escolares (mediante interrogaciones por criterios temáticos que decide el usuario), localización del domicilio (el sistema facilita utilidades para buscar la parcela catastral), cálculo de distancias y baremación y visualización de resultados.

Los usuarios interaccionan con el geo servicio cuando facilitan los parámetros de búsqueda de los centros escolares y proporcionan al sistema la información necesaria para que éste localice la parcela catastral del domicilio (Figura 4). En consecuencia, este geo servicio se puede clasificar en la fase 3 de integración, según el modelo de clasificación que seguimos. 
Tabla 1. Resumen de las características del Buscador de Centros Educativos del Gobierno de Aragón.

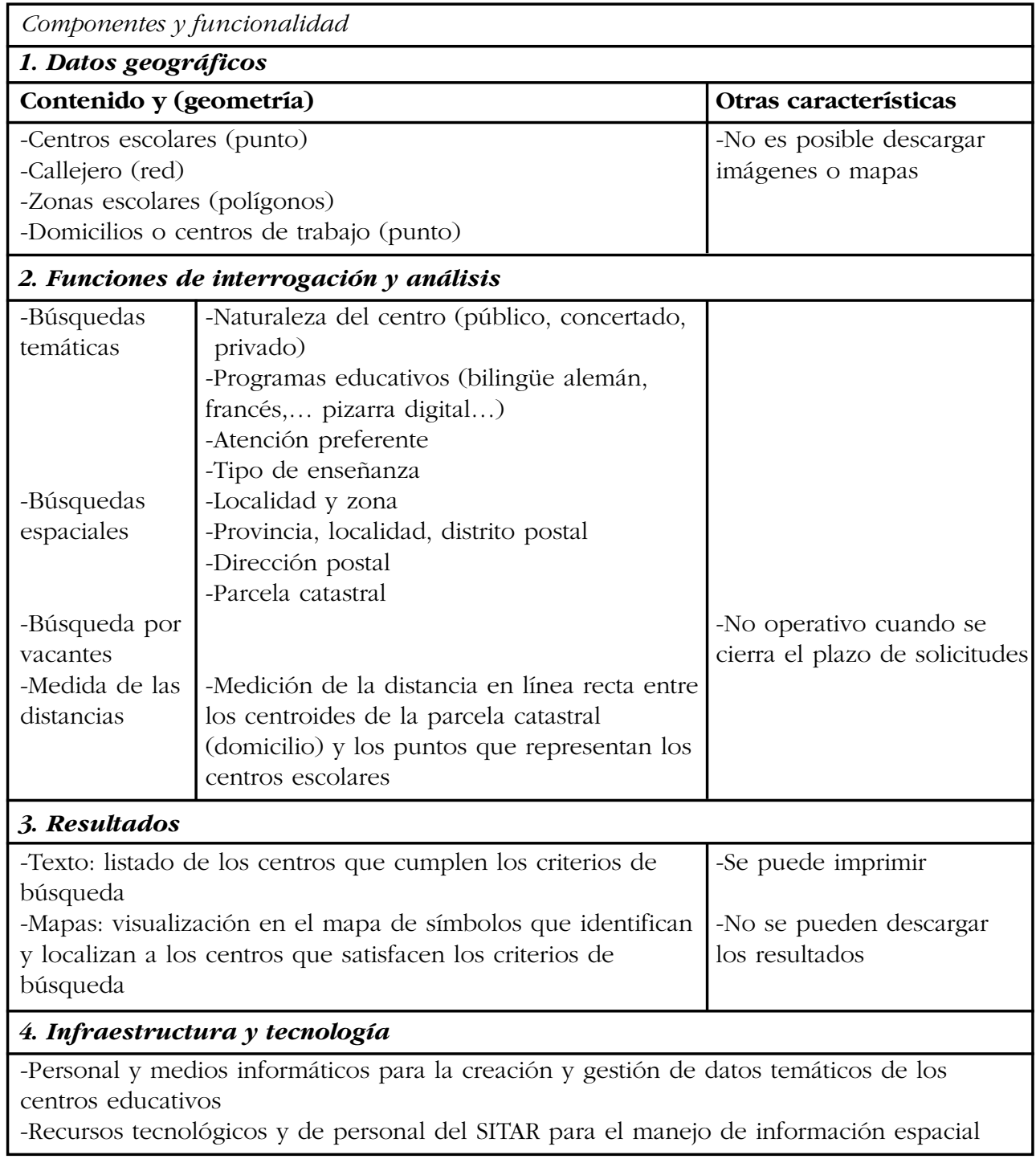

Fuentes: Faci (2010) y Gobierno de Aragón: http://www.centroseducativosaragon.es/Public/default.aspx (Consulta: 15.10.2010). Elaboración propia. 


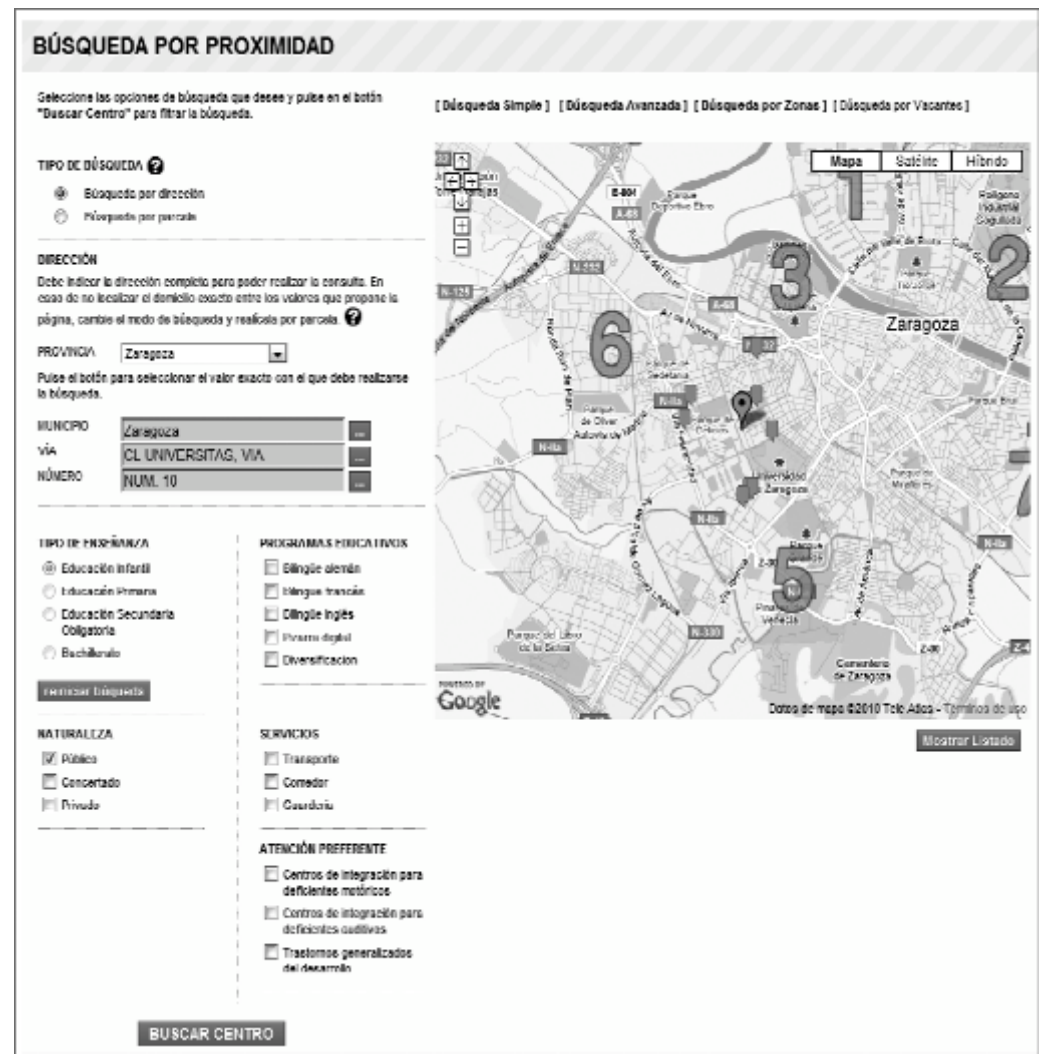

Figura 4. Interface de búsqueda por proximidad.

Fuente: http://www.centroseducativosaragon.es/Public/buscador_centros_listado.aspx (Consulta: 15.10.2010).

\subsection{Geo servicios en IDEZar (Infraestructura de datos espaciales de Zaragoza)}

La información geográfica de la infraestructura de datos espaciales de Zaragoza ha multiplicado su uso y utilidad con la incorporación al sistema de recursos (gadgets) para el manejo y visualización de la información en varios canales (Internet, telefonía móvil, GeoRSS) y de otras aplicaciones más complejas que son propiamente geo servicios, de acceso público o bien para uso interno (planificación de eventos de policía local, gestión de incidencias sobre la vía pública, gestión de medios y recursos de bomberos y protección civil para el plan de emergencias) (Pérez-Pérez, Rodrigo et al., 2009; Fernández, Pérez-Pérez et al., 2010). 
Parte de los geo servicios públicos están dedicados a proporcionar información temática y de la localización de las instalaciones y dependencias municipales, de diversos contenidos del estado del medio ambiente o sobre incidencias en vía pública. En su mayoría se encuadran dentro de la fase 1 del modelo que aquí manejamos. Otros, son más avanzados y permiten la interacción con los usuarios que deben facilitar ciertos valores de parámetros (temáticos, espaciales y temporales) que se utilizan para llevar a cabo determinados cálculos: tal es el caso del callejero, el buscador de rutas de llegada a Zaragoza, o el planificador de itinerarios turísticos en la ciudad de Zaragoza. Estos últimos se encuadran en la fase 3 del modelo referido (tabla 2; figura 5).

Tabla 2. Resumen de las características del IDEZar.

Componentes y funcionalidad

\section{Datos geográficos}

\begin{tabular}{|l|l}
\hline Contenido y (geometria) & Servicios
\end{tabular}

-Servicios del Ayuntamiento (punto)

-Incidencias en vía pública (puntos)

-Callejero (red)

-Información medioambiental (puntos,líneas

y polígonos)

-Otras clases de elementos (manzanas urba-

nas, delimitaciones administrativas...)

-Acceso a información de otras geo bases

-Servicio de mapas en Internet (WMS) (mapa base de Zaragoza, mapas temáticos, cálculo de rutas, mapa temático de medio ambiente -GeoRSS (cortes de agua, cortes de tráfico, otras afecciones importantes, antena móviles)

-Servicios para mashups (Puntos de interés)

-Servicio de acceso a datos (SOAP)) (Callejero)

-Nomenclátor (de la IDE Inst. Geográfico nacional)

\section{Geo Servicios públicos}

\begin{tabular}{|l|l}
\hline Callejero & El usuario selecciona el nom-
\end{tabular} bre de la vía y la clase de información urbana en un panel desplegable con 14 categorías. El programa ofrece información detallada sobre la calle seleccionada y ayuda en las búsquedas ambiguas
La aplicación muestra los resultados mediante símbolos en un mapa cuya base son las manzanas urbanas las calles con el nombre, espacios verdes y no construidos y la red hidrográfica. Se pueden añadir también las parcelas catastrales. El mapa se acompaña de herramienta ayudar a la visualización (volver al mapa inicial, volver a la vista anterior, ir al mapa siguiente, mostrar y ocultar un mapa de situación,, mover el mapa, acercar el mapa, alejar el mapa, mostrar la leyenda) imprimir el mapa, obtener ayuda y mostrar la leyenda. Y sobreponer el plano catastral. 
Componentes $y$ funcionalidad

\section{Geo Servicios públicos}

\begin{tabular}{l|l}
\hline Planos & Colección de mapas con la localización de determinadas categorías de obje-
\end{tabular}

temáticos

Localización

de centros

municipales

Zaragoza

desde el aire

Incidencias

en vía

pública

Cómo llegar

tos. Se facilitan herramientas de navegación en el mapa

Clasificación de los servicios municipales que se presenta en paneles desplegables para seleccionar por el usuario. Se puede explorar su localización en un mapa. Se facilita herramientas de navegación en el mapa

Visualiza la ciudad de Zaragoza de la imagen de Google Earth@.

Se muestra un mapa con las incidencias en vía pública correspondientes a: cortes de agua, cortes de tráfico, afecciones importantes

Este geo-servicio calcula y visualiza la ruta más rápida o más corta de cualquier localidad de España a Zaragoza. El usuario selecciona el tipo de ruta y la localidad

Márgenes $\quad$ Se muestran, sobre una ortofoto los proyectos de márgenes y riberas urbanas y riberas del río Ebro en la ciudad de Zaragoza. De cada proyecto se muestra, además información sobre su contenido y estado actual así como varias imágenes.

Medio

ambiente Se muestra información en un mapa con medidas de los niveles de inmisón en el aire de distintas sustancias, medidos en las estaciones de la red de control dela contaminación atmosférica. También contiene un mapa de localización del Galacho de Juslibol, que se completa con abundante documentación

Estos geo servicios disponen de herramientas para navegar por los mapas y la imagen: acercar, alejar (cambio de escala), mover el mapa y volver a la vista original.

Planifica tu $\quad$ Esta aplicación busca recursos visita turisticos de acuerdo a las preferencias del usuario. Éste puede seleccionar diferentes opciones predeterminadas en 5 categorías temáticas (motivos de la estancia, compañía, preferencias, alojamiento y otros) y el día de la visita. La interfaz puede ampliarse con otras opciones de 4 nuevas categorías temáticas (estilos artísticos, tipo de comida no me interesan y más preferencias) y la selección de monumentos que se desean visitar
La ruta propuesta presenta información con el itinerario de los lugares a visitar y el tiempo estimado de la visita; el usuario puede desechar algunos lugares y el sistema recalcula la ruta. Además, se muestra una imagen de cada lugar o monumento y datos sobre los horarios de visita. El documento se completa con la ubicación de cada lugar en un mapa de Google Maps (C).En la misma página se tiene acceso a otra información relacionada con el turismo

Fuente: http://www.zaragoza.es/ciudad/idezar/index.htm (Consulta: 20.10.2010). Elaboración propia. 
La infraestructura de datos geográficos IDEZar constituye una buena plataforma para la creación de aplicaciones de geo servicios avanzados, tanto para los departamentos del propio Ayuntamiento como para las empresas.

\section{PLANIFICA TU VISITA}

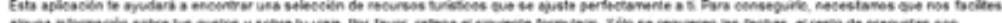

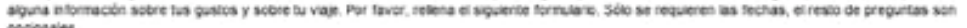

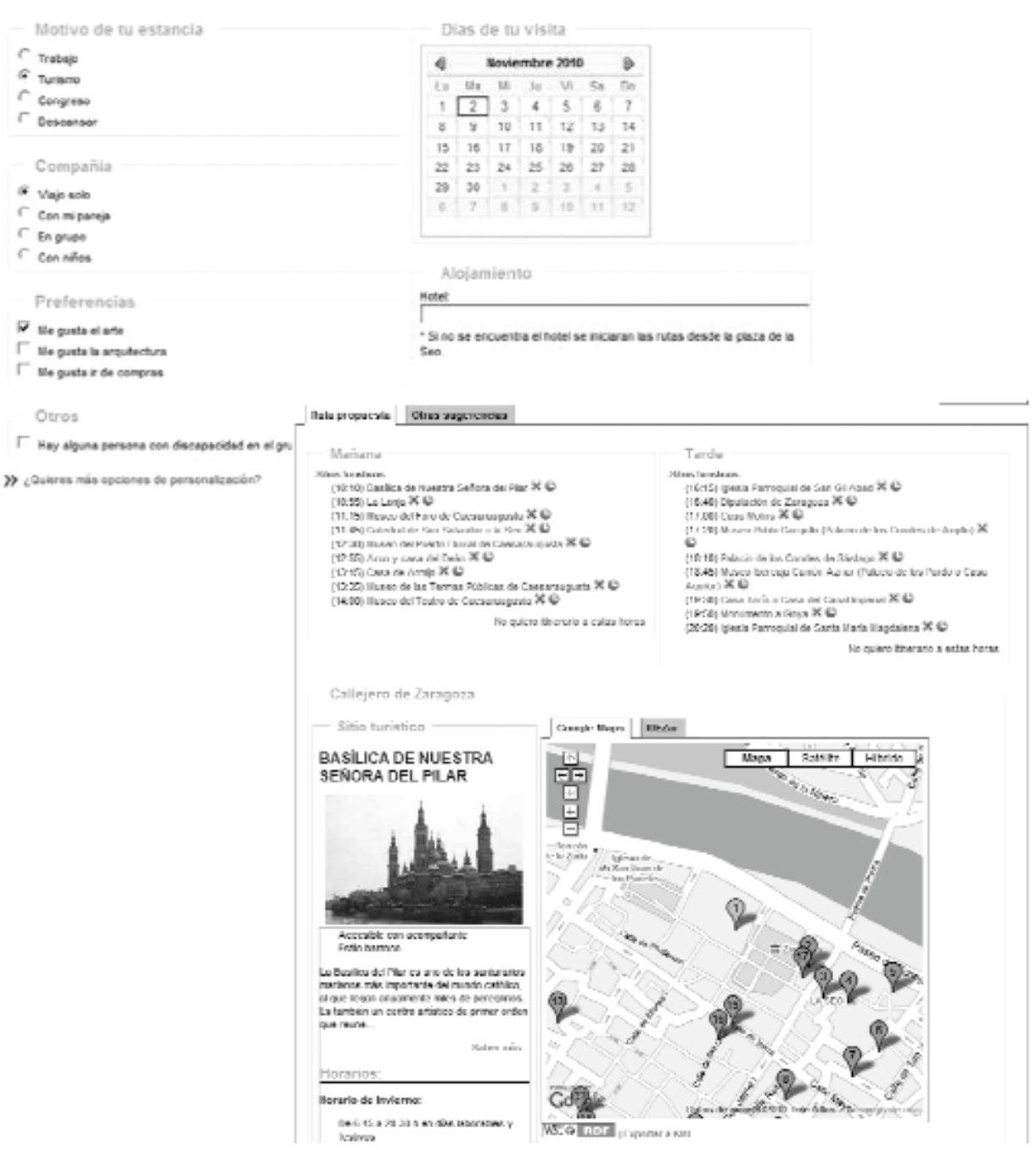

Figura 5. Interfaz y resultados del geo servicio planificador de itinerarios turísticos

Fuente: http://www.zaragoza.es/ciudad/idezar/index.htm (Consulta: 20.10.2010). 


\section{Consideraciones finales}

La administración autonómica y local aragonesa no es una excepción en cuanto a la debilidad de geo servicios en todos los niveles de la administración pública española.

El desarrollo de los geo-servicios, públicos y privados, está muy condicionado por la disponibilidad de información geográfica con la resolución y formatos adecuados. Otro factor limitante lo constituyen las elevadas exigencias de las infraestructuras y equipamiento necesarios para la gestión y trasmisión de la información geográfica, así como la complejidad inherente a ciertas operaciones de análisis espacial. Pero, tal vez, la causa principal del modesto desarrollo y de los geo servicios en la administración pública se encuentre en la fragmentación de los departamentos y servicios y la inveterada tendencia a su funcionamiento aislado.

Estas razones pueden explicar también las fases embrionarias de integración en que se encuentran los geo-servicios existentes.

Los geo-servicios examinados han recibido numerosas consultas y han sido valorados positivamente por los usuarios, a pesar de las limitadas opciones de interacción que ofrecen. Esta y otras experiencias avalan la creación y difusión de nuevos geoservicios en el marco del desarrollo de la administración electrónica.

El marco legal así como la información geográfica y las infraestructuras técnicas existentes en Aragón permiten que las administraciones públicas elaboren nuevos geo-servicios. Son bastantes las áreas en las que su aplicación resultaría beneficiosa para los ciudadanos y para la propia administración, en especial, en la organización de diversos servicios de emergencia, la prevención de riesgos de todo tipo, la adscripción de usuarios a ciertos equipamientos públicos y otras.

Sin embargo, la utilidad social de los geo-servicios que se lleguen a crear pasa por la necesaria cooperación entre los departamentos y servicios de la Administración implicados en su gestión y los usuarios a los que van dirigidos. La colaboración es más factible en el marco de la construcción de la administración electrónica, concebida como una de las vías para la necesaria modernización de la administración. 


\section{Bibliografía}

Álvarez, P., P. Muro, et al. (2006). IDEZar: un ejemplo de implantación de una IDE en la Administración Local. IX Jornadas sobre Tecnologías de la Información para la Modernización de las Adminsitraciones Públicas (TECNIMAP), Sevilla.

Ayuntamiento de Zaragoza-Web Municipal. (2010). "Infraestructuras de datos espaciales de Zaragoza (IDEZAR)". Consultada 4 octubre 2010, de http://www.zaragoza.es/ ciudad/idezar/index.htm.

Bhatnagar, S. C. (2004). E-government: from vision to implementation: a practical guide with case studies. New Delhi; Thousand Oaks, Calif., Sage Publications.

Comisión Europea (2003). The Role of eGovernment for Europe's Future. Bruselas. URL: http://ec.europa.eu/information_society/eeurope/2005/doc/all_abo ut/egov_communication_en.pdf (Consulta: 08.09.2010).

Chaín, C. and P. Tomás (2005). Análisis comparativo del desarrollo en Internet de los municipios españoles más poblados. Anales de Documentación, 8: 37-49.

Esteves, J. (2006). Análisis del desarrollo del gobierno electrónico municipal en España. Revista de Empresa, 15: 86-96.

European Commission INSPIRE. Consultada 4 octubre 2010, de http://inspire.jrc.ec. europa.eu/index.cfm.

Faci, F. (2010). Nuevo criterio de proximidad en la elcción de centro basado en la geolocalización. XI Jornadas sobre Tecnologías de la Información para la Modernización de las Administraciones Públicas, Zaragoza, 6-9 de abril.

Fernández, M. J., M. J. Pérez-Pérez, et al. (2010). IDEZar 2.0: interacción, usabili- dad y accesibilidad. TECNIMAP. Zaragoza, TECNIMAP.

Fountain, J. E. (2001). Building the virtual state: information technology and institutional change. Washington, D.C., Brookings Institution Press.

Garson, G. D. (2000). Social dimensions of information technology: issues for the new millennium. Hershey; London, Ideas Group.

Gobierno de Aragón. (2010). "Departamento de Educación, Cultura y Deporte. Portal de Centros Educativos." de http:// www.centroseducativosaragon.es/Public/ default.aspx.

Gobierno de Aragón (s/f a). I Plan Director de Infraestructuras de Telecomunicaciones en Aragón, Departamento de Ciencia, Tecnología y Universidad.

Gobierno de Aragón (s/f b). Plan de Administración Electrónica del Gobierno de Aragón. D. d. Presidencia. Zaragoza, Gobierno de Aragón.

Gobierno de Aragón (s/f c). Plan Director para el desarrollo de la sociedad de la información en la Comunidad Aragón 2.0 .

Grönlund, A. A. (2001). Electronic government: design, applications and management. Hershey, PA, Idea Group Publishing.

Grönlund, A. A. (2004). State of the Art in EGov Research. A survey. EGOV 2004, Heidelberg, Springer.

Mas, S., A. García, et al. (2010). CartoCiudad: Una apuesta colaborativa de las Administraciones Públicas en el ámbito d elos callejeros. XI Jornadas sobre 
Tecnologías de la Información para la Modernización de las Administraciones Públicas, Zaragoza, 6-9 de abril.

Millard, J., J. Shahin, et al. (2009). i2010 eGovernment Action Plan. Progress study (SMART 2008/0042). Final Report. Bruselas, European Commisison. Information Society and Media.

Osborne, D. and T. Gaebler (1992). Reinventing government: how the entrepreneurial spirit is transforming the public sector. Reading, Mass., Addison-Wesley Pub. Co.

Pérez-Pérez, M. J., P. Rodrigo, et al. (2009). IDEZar 2.0 para la Administración y Gestión de Datos Espaciales de España JIDEE, Murcia.

Portolés-Rodríguez, D., P. Álvarez, et al. (2005). IDEZar: an Example of User Needs, Technological Aspects and the International Framework of a Local SDI. 11th EC-GI\&GIS Workshop: ESDI: Setting The Framework, Alguero, Cerdeña.

Rivera, E. (2003). Nueva economía, gobierno electrónico y reforma del Estado. Chile a la luz de la experiencia internacional.
Santiago, FLACSO-chile, Editorial Universitaria.

Scholl, H. J. (2009). Profiling the EG research community and Its core. 8th International Conference, EGOV 2009, Linz, Austria, Springer.

Tapscott, D. (1996). The digital economy: promise and peril in the age of networked intelligence. New York ; London, McGrawHill (Traducción: la economía digital, 1997, MCGraw-Hill, bogotá).

Traunmüller, R. and M. A. Wimmer (2005) Online One-stop Government 47 (2005), 10, Nr. 5, S. 384. Wirtschaftsinformatik, 47(5): 384

Wimmer, M. A. (2001). European Development Towards Online One-stop government: The "eGOV" Project. ICEC, viena.

Wimmer, M. A., R. Traunmüller, et al. (2001). Electronic Business Invading the Public Sector: Considerations on Change and Design Procedings of the 34th Annual Hawaii International Conference on System Siciences (HICSS-34), Maui, Hawaii, Institute of Electrical and Electronics Engineers Inc. 